\title{
How to Steal a Botnet and What Can Happen When You Do
}

\section{Richard A. Kemmerer}

\author{
Security Group \\ Department of Computer Science \\ University of California, Santa Barbara \\ kemm@cs.ucsb.edu \\ (2) UC SANTA BARBARA \\ engineering
}




\section{Botnet Terminology}

UC Santa Barbara

- Bot

- an application that performs some action or set of actions on behalf of a remote controller

- installed on a victim machine (zombie)

- modular (plug in your functionality/exploit/payload)

- Botnet

- network of infected machines controlled by a malicious entity

- Control channel

- required to send commands to bots and obtain results and status messages

- usually via IRC, HTTP, HTTPs, or Peer-to-Peer

- Bot Herder

- aka botmaster or controller

- owns control channel, sends commands to botnet army

- motivations are usually power or money 


\section{Torpig}

- Trojan horse

- distributed via the Mebroot "malware platform"

- injects itself into 29 different applications as DLL

- steals sensitive information (passwords, HTTP POST data)

- HTTP injection for phishing

- uses "encrypted" HTTP as C\&C protocol

- uses domain flux to locate C\&C server

- Mebroot

- spreads via drive-by downloads

- sophisticated rootkit (overwrites master boot record) 
Torpig: Behind the scenes

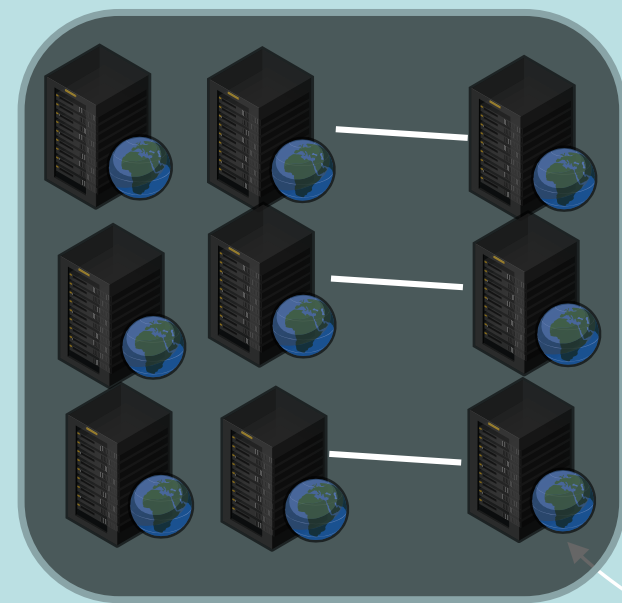

"Hacked" web servens

;앙

;3)

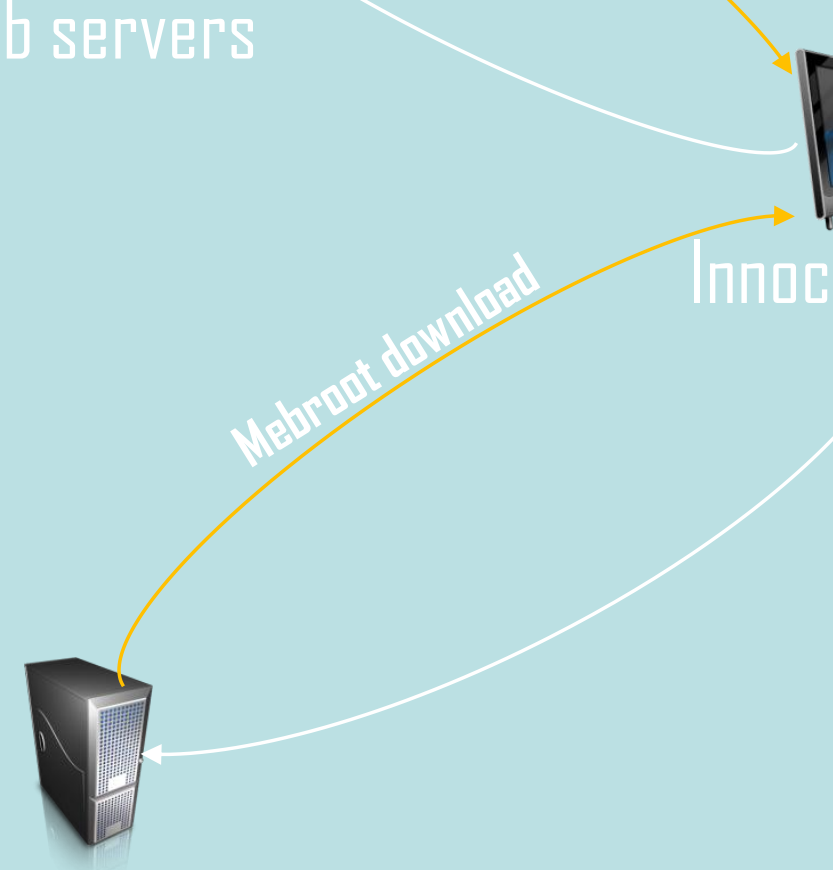

"Drive-By Dawnladed" server
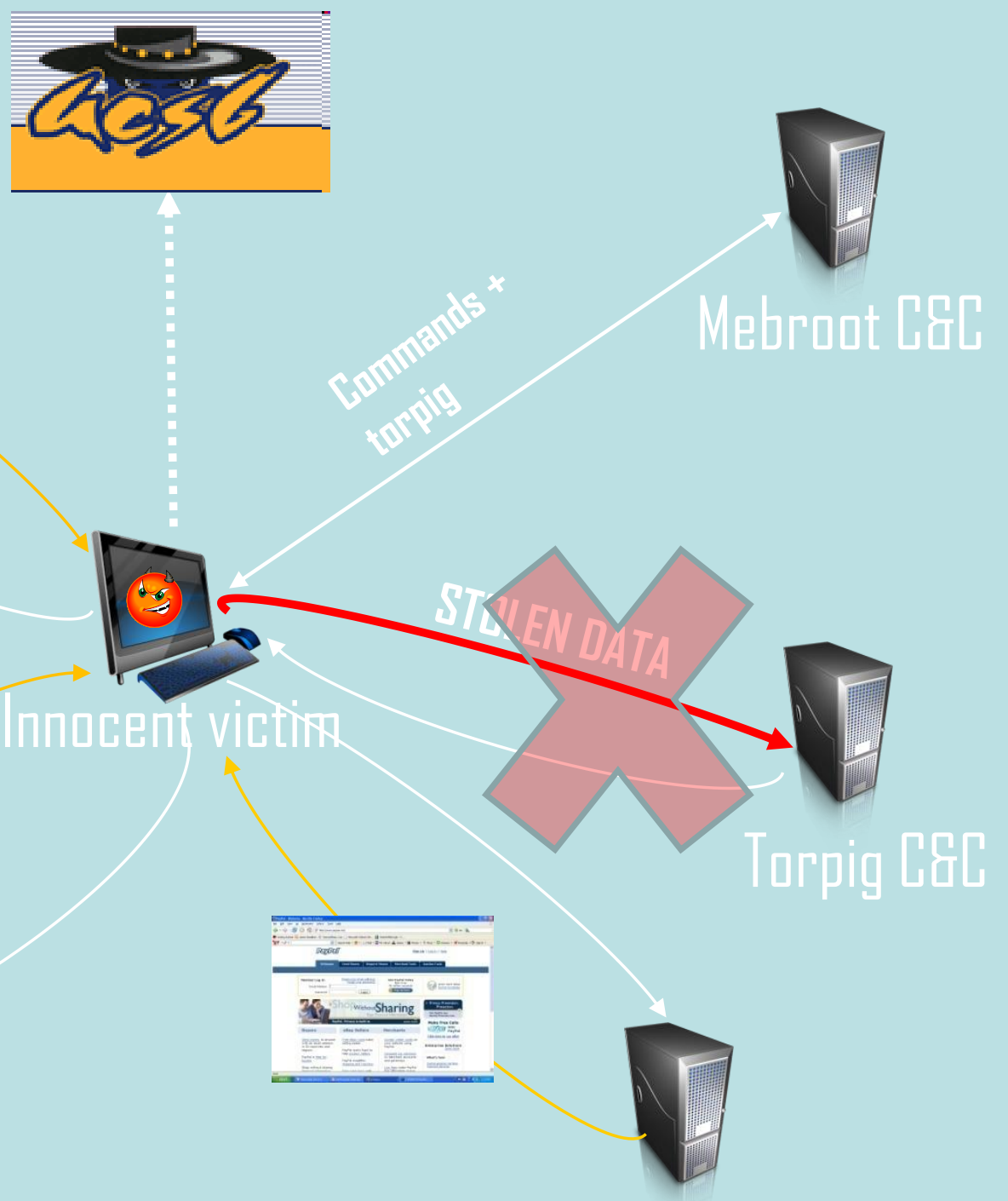

Injection server 


\section{Torpig HTML Injection}

UC Santa Barbara

- Domains of interest ( 300) stored in configuration file

- When domain of interest visited

- Torpig issues request to injection server

- server specifies a trigger page on target domain and a URL on injection server to be visited when user visits trigger page

- When user visits the trigger page

- Torpig requests injection URL from injection server

- Torpig injects the returned content into the user's browser

- Content is usually html phishing form that asks for sensitive data

- reproduces look and style of target web site 


\section{Example Phishing Page}

UC Santa Barbara

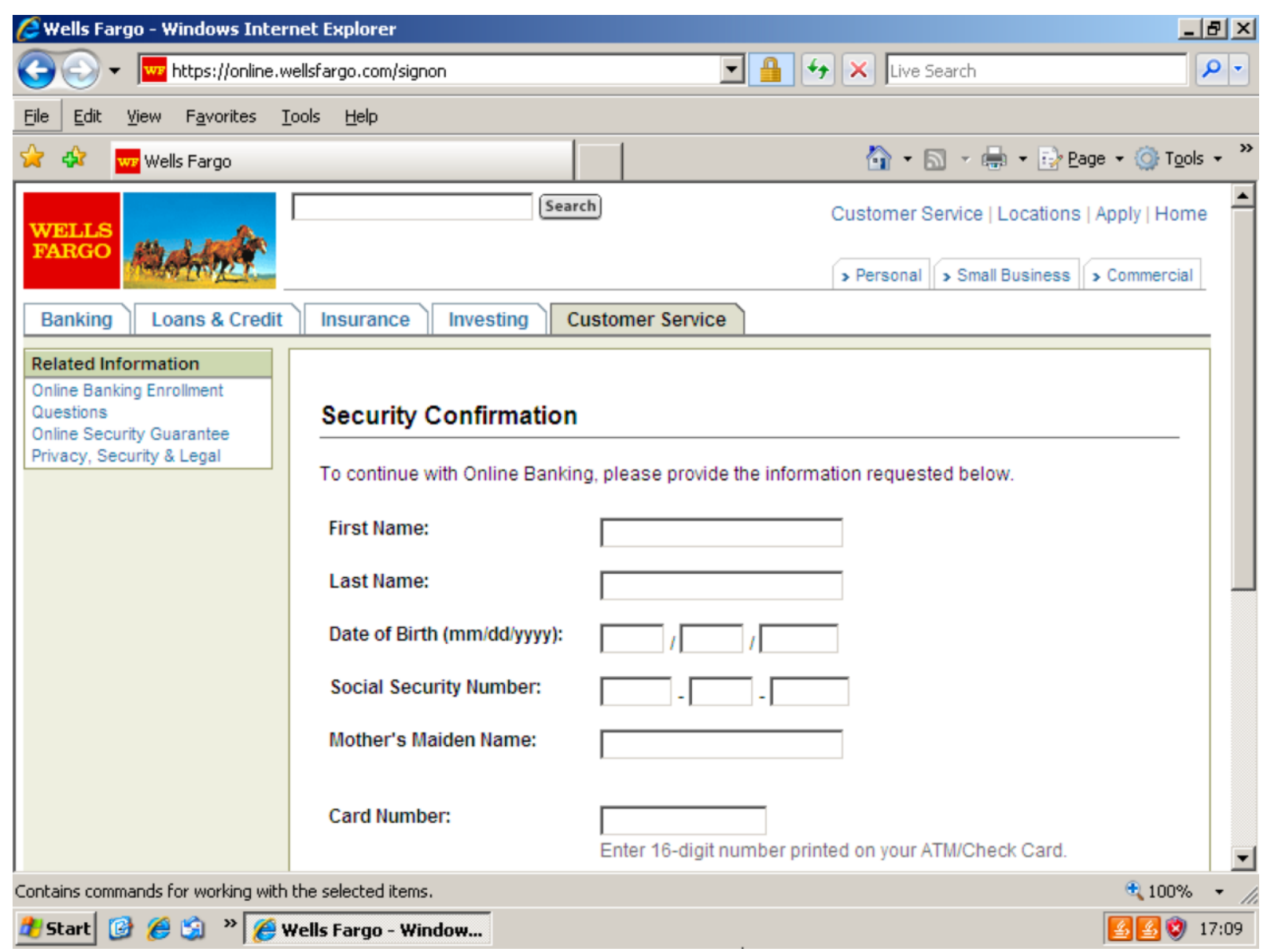




\section{Example Phishing Page}

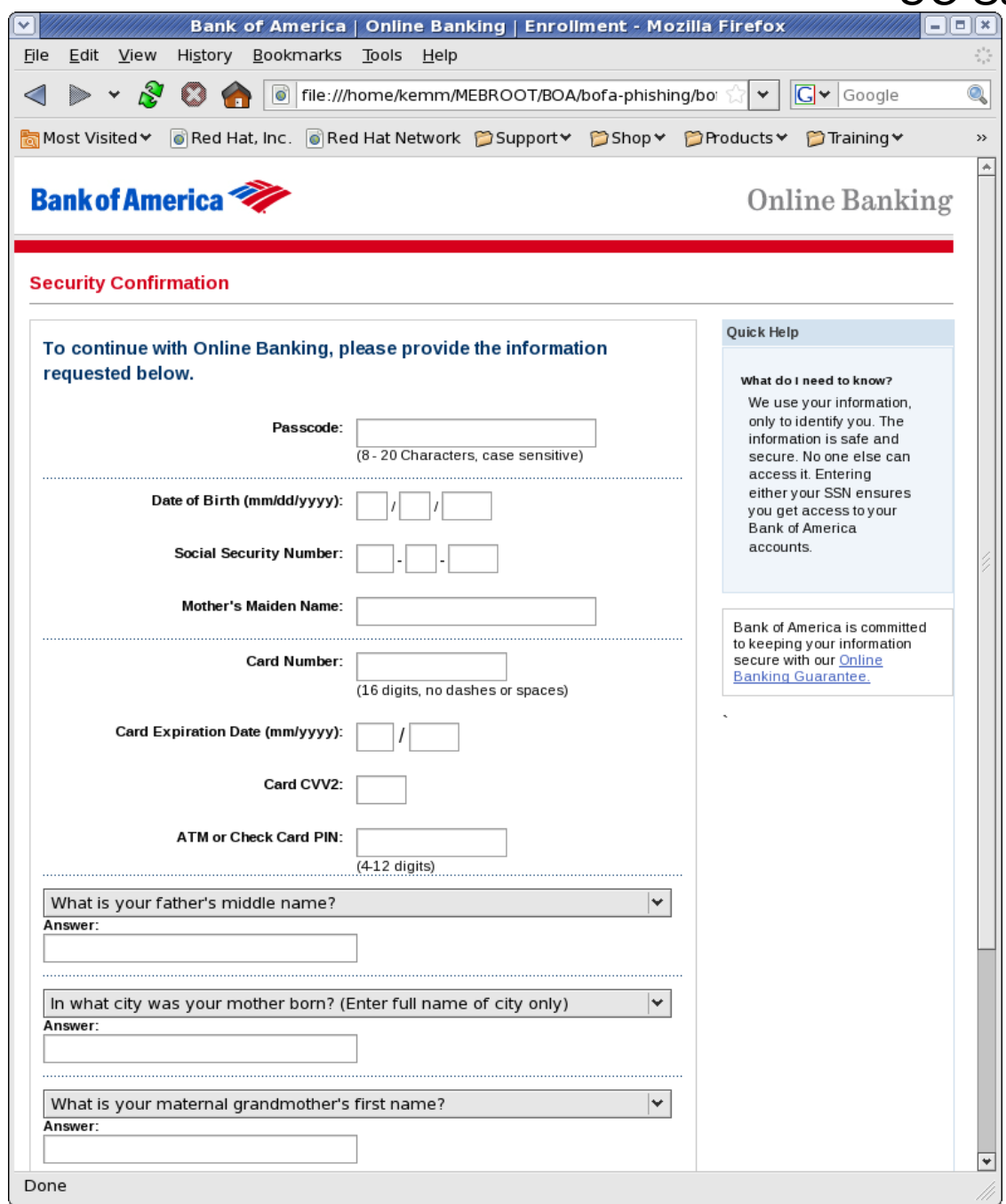




\section{Domain Flux}

- Taking down a single bot has little effect on botmaster

- C\&C servers are vulnerable to take down

- if you use a static IP address, people will block or remove host

- if you use a DNS name, people will block or remove domain name

- Domain flux

- idea is to have bots periodically generate new C\&C domain names

- often, use local date (system time) as input

- botmaster needs to register one of these domains and respond properly so that bots recognize valid C\&C server

- defenders must register all domains to take down botnet 


\section{Torpig Domain Flux}

UC Santa Barbara

- Each bot has

- same domain generation algorithm (DGA)

- three fixed domains to be used if all else fails

- DGA generates

- weekly domain name (wd)

- daily domain name (dd)

- Every 20 minutes bot attempts to connect (in order) to

- wd.com, wd.net, wd.biz

- if all three fail, then dd.com, dd.net, dd.biz

- if they also fail, then the three fixed domains

- Criminals normally registered wd.com (and wd.net) 


\section{Sinkholing Torpig C\&C Overview}

UC Santa Barbara

- Reverse engineered name generation algorithm and C\&C protocol

- Observed domains for 01/25 - 02/15 unregistered

- Registered these domains ourselves

- Unfortunately, Mebroot pushed new Torpig binary on 02/04

- We controlled the botnet for $\sim 10$ days

- Data

- 8.7 GB Apache logs

- 69 GB pcap data (contains stolen information) 


\section{Sinkholing Torpig C\&C}

UC Santa Barbara

- Purchased hosting from two different hosting providers known to be unresponsive to complaints

- Registered wd.com and wd.net with two different registrars

- One was suspended 01/31 due to abuse complaint

- Set up Apache web servers to receive bot requests

- Recorded all network traffic

- Automatically downloaded and removed data from our hosting providers

- Enabled hosts a week early

- immediately received data from 359 infected machines 


\section{Data Collection Principles}

UC Santa Barbara

- Principle 1: the sinkholed botnet should be operated so that any harm and/or damage to victims and targets of attacks would be minimized

- always responded with okn message

- never sent new/blank configuration file

- removed data from servers regularly

- stored data offline in encrypted form

- Principle 2: the sinkholed botnet should collect enough information to enable notification and remediation of affected parties

- worked with law enforcement (FBI and DoD Cybercrime units)

- worked with bank security officers

- worked with ISPs 


\section{Data Collection}

- Bot connects to Torpig C\&C every 20 minutes via HTTP POST

- Sends a header

- timestamp, IP address, proxy ports, OS version, locale, nid, Torpig build and version number

- nid

- 8 byte value, used for encrypting header and data

- derived from hard disk information or volume serial number

- serves as a convenient, unique identifier

- allows one to detect VMware machines

- Optional body data

- stolen information (accounts, browser data, ...) 


\section{Size Estimation}

UC Santa Barbara

- Count number of infections

- usually based on unique IP addresses

- problematic: DHCP and NAT effects (we saw 1.2M unique IPs)

- our count based on header information: $180 \mathrm{~K}$ hosts (nids) seen
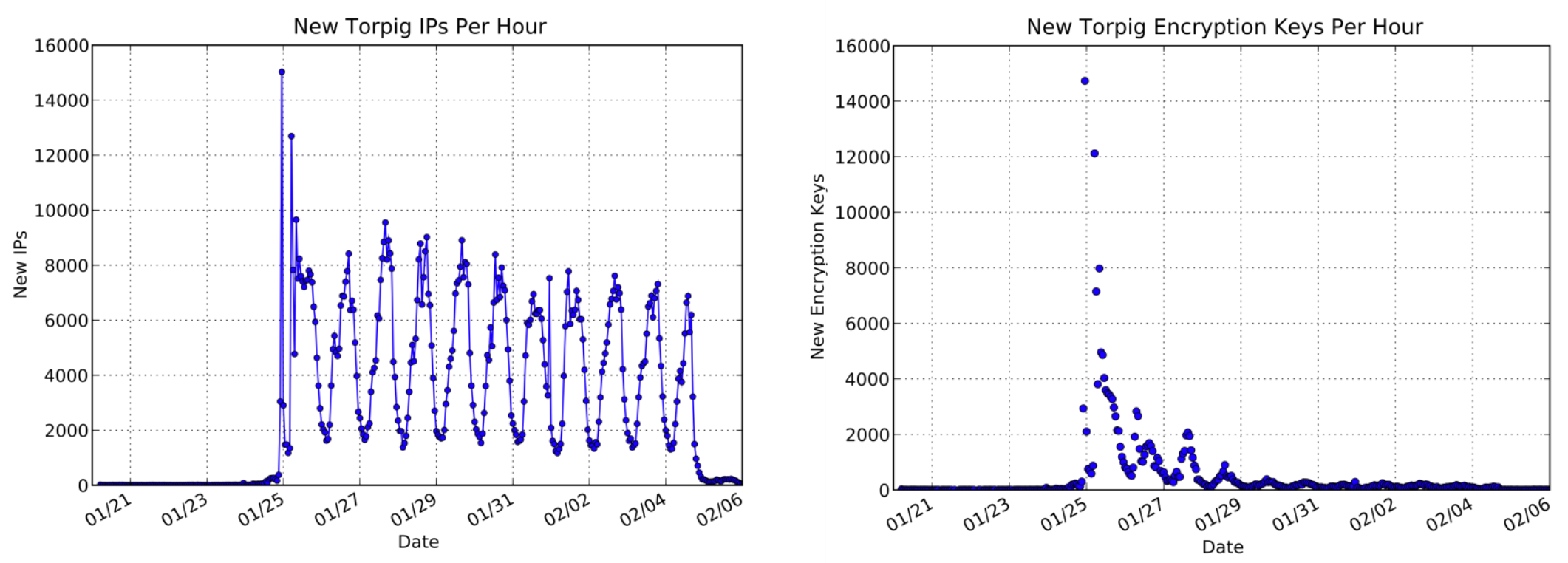

Average 4,690 new IPs

Average 705 new nids 


\section{Size Estimation}

UC Santa Barbara

- Cummulative number of infections

- linear for unique IP addresses

- decayed quickly for unique nids

- more than $75 \%$ of unique nids were observed in first 48 hours
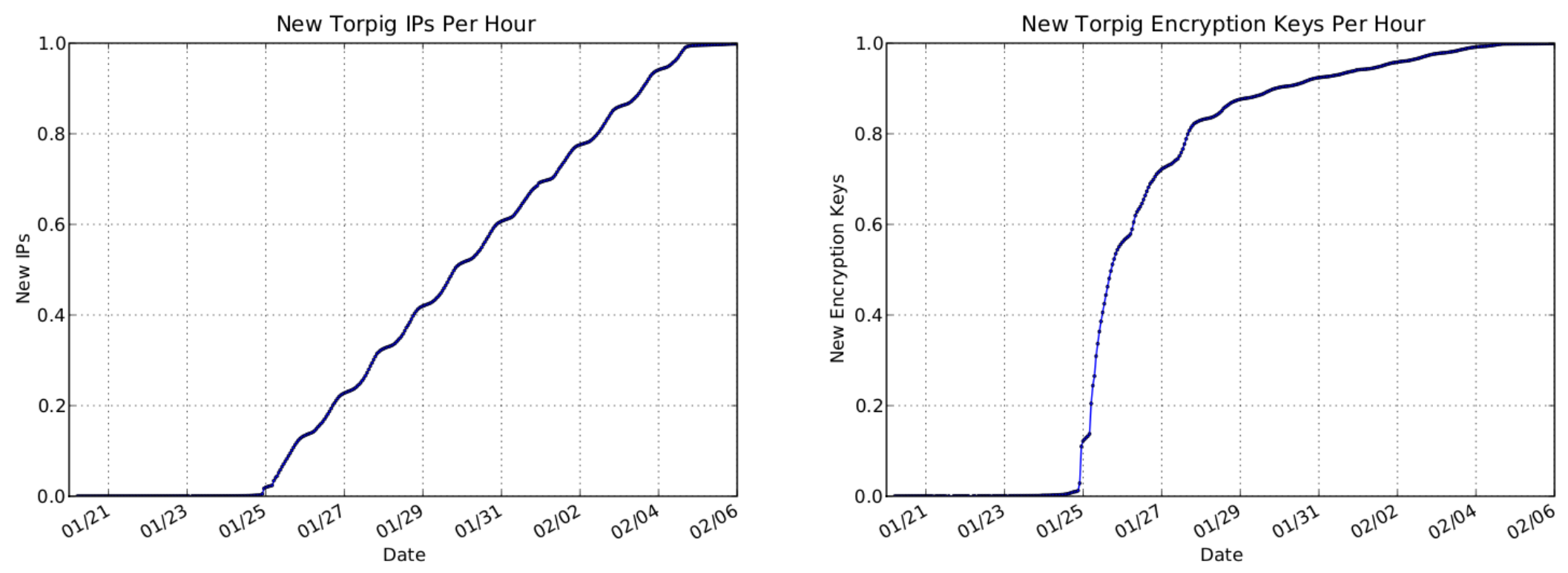


\section{Threats}

- Theft of financial data

- Denial of service

- Proxy servers

- Privacy threats 


\section{Threats: Theft of Financial Information}

- 8,310 unique accounts from 410 financial institutions

- Top 5: PayPal $(1,770)$, Poste Italiane, Capital One, E`Trade, Chase

- $38 \%$ of credentials stolen from browser's password manager

- 1,660 credit cards

- Top 5: Visa $(1,056)$, Mastercard, American Express, Maestro, Discover

- US (49\%), Italy (12\%), Spain (8\%)

- typically, one CC per victim, but there are exceptions ... 


\section{Value of the Financial Information}

UC Santa Barbara

- Symantec [2008] estimates

- Credit card value at $\$ .10$ to $\$ 25.00$

- Bank account at $\$ 10.00$ to $\$ 1,000.00$

- Using Symantec estimates,10 days of Torpig data valued at $\$ 83 \mathrm{~K}$ to $\$ 8.3 \mathrm{M}$

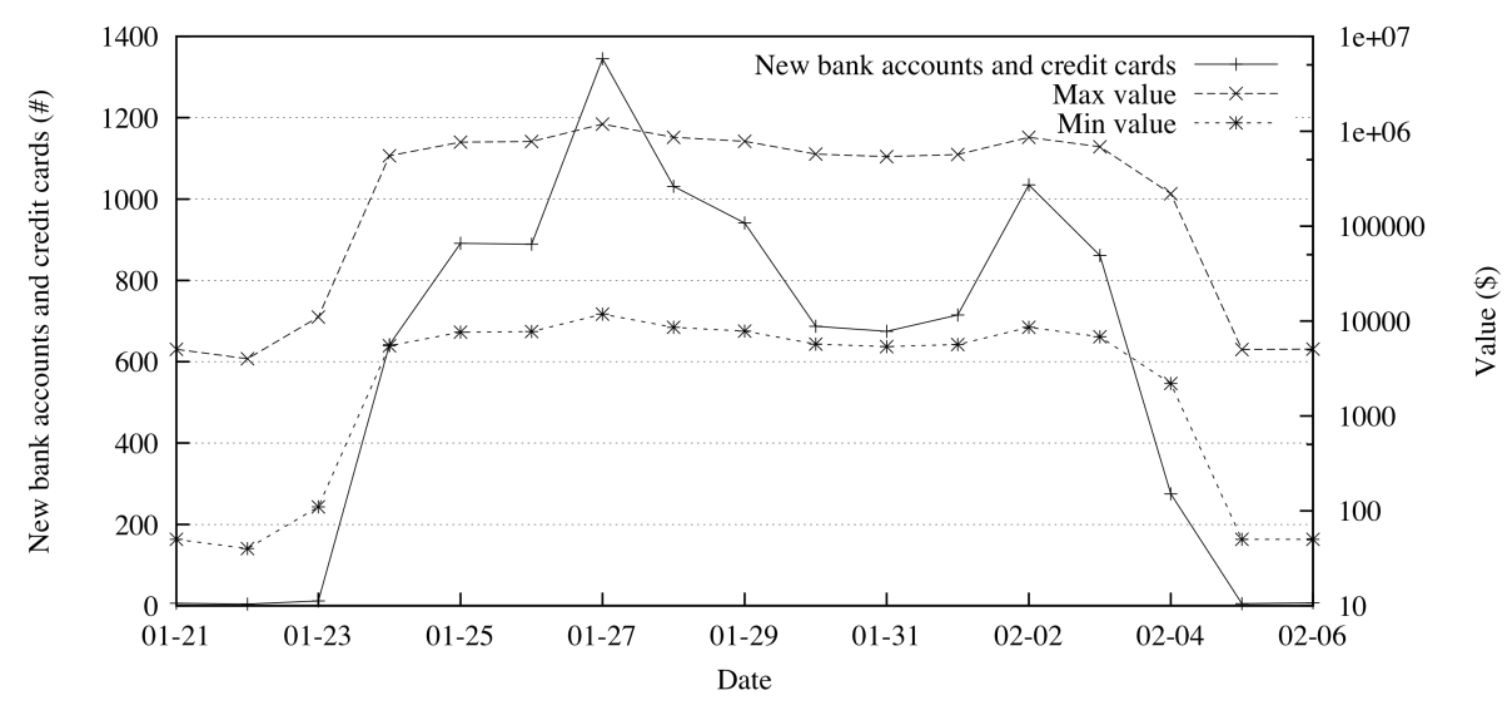




\section{Threats: Denial of Service}

- More than 60,000 active hosts at any given time

- Determine network speed from ip2location DB

- cable and DSL make up $65 \%$ of infected hosts

- used 435 kbps conservative upstream bandwidth

- yields greater than 17 Gbps just from DSL/cable

- corporate networks make up $22 \%$ of infected hosts

- Potential for a massive DDOS attack 


\section{Threats: Proxy Servers}

UC Santa Barbara

- Torpig opens SOCKS and HTTP proxy

- $20 \%$ of infected machines are publicly reachable

- Only $2.45 \%$ of those marked by Spamhaus blacklist

- Could be abused for spamming 


\section{Threats: Privacy}

- Web mail, web chat, and forum messages

- Focused on 6,542 messages in English that were 250 characters or longer

- Zeitgeist of the Torpig network

- $14 \%$ are about jobs/resumes

- $7 \%$ discuss money

- $6 \%$ are sports fans

- $5 \%$ prepare for exams and worry about grades

- 4\% partners/sex online

- Online security is a concern, but think they are clean

- $10 \%$ specifically mention security/malware 


\section{Password Analysis}

UC Santa Barbara

- 297,962 unique credentials used on 368,501 web sites (domains)

- mostly web mail (Google, live, Yahoo) and social networking sites (Facebook, MySpace, netlog.com)

- $28 \%$ of the victims reused their password on multiple domains

- Used John the Ripper to assess the strength of the passwords

- 173,686 unique passwords

- 56,000 in < 65 minutes using permutation, substitution, etc.

- 14,000 in next 10 minutes using large wordlist

(i.e., $40 \%$ cracked in less than 75 minutes)

- another 30,000 in next 24 hours 


\section{Password Analysis}

UC Santa Barbara

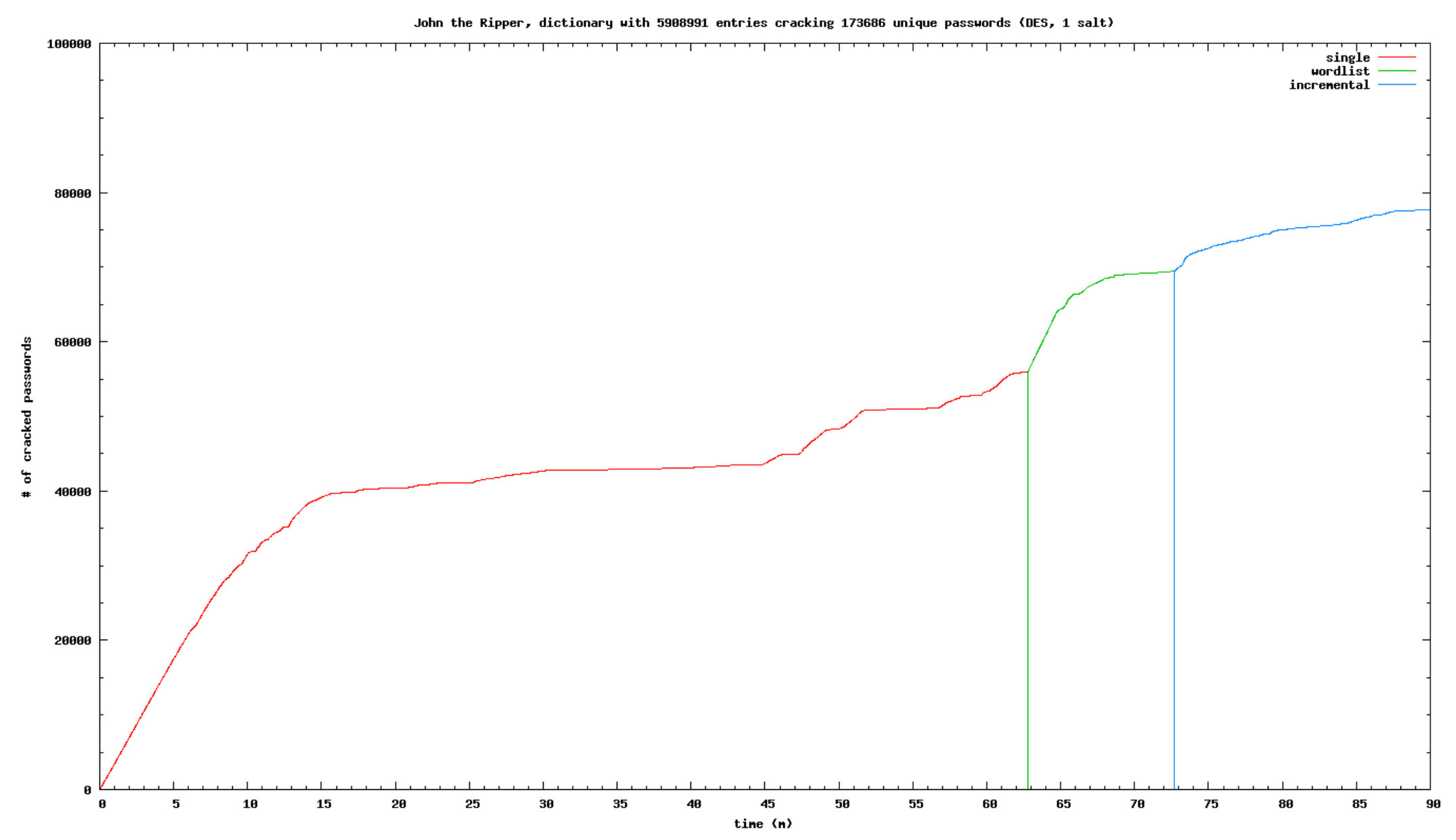




\section{What about?}

- Criminal retribution

- Law enforcement

- Repatriating the data

- Ethics, IRB, etc. 


\section{Criminal Retribution}

UC Santa Barbara

- Big concern on January 25

- are the criminals going to come to get us?

- More realistically - when will they DDOS our servers?

- Biggest question - why did it take them 10 days to download a new DGA? 


\section{Law Enforcement}

UC Santa Barbara

- We needed to inform law enforcement about this

- who do we notify?

- need someone knowledgeable so they don't shut us down

- How do we get a hold of law enforcement?

- US CERT gives you a form to fill out

- contacted David Dagon at Ga Tech and got FBI contact

- contacted FBI cybercrime unit

- also contacted DoD defense criminal investigative services

- FBI was very good to work with and gave us lots of contacts for repatriation 


\section{Repatriating the Data}

UC Santa Barbara

- 8,310 accounts from 410 financial institutions

- 1,660 credit cards from various financial institutions

- Need to mine the information from the raw data files

- Cannot just cold call a bank and say I have information that you might want, send me your BINs

- Need introductions from trusted individuals or groups

- FBI and National Cyber-Forensics and Training Alliance (NCFTA) were very helpful

- leads to individuals who could handle an entire country 


\section{Ethics}

- Recall Principle 1: the sinkholed botnet should be operated so that any harm and/or damage to victims and targets of attacks would be minimized

- Collected sensitive data that potentially could threaten the privacy of victims

- Should emails be viewed at all?

- What about IRB approval?

- not working with human subjects, why would we need it?

- we didn't plan on getting this kind of data

- any data that can be used to identify an individual needs IRB 


\section{Conclusions}

UC Santa Barbara

- Unique opportunity to understand

- potential for profit and malicious activity of botnet's creators

- characteristics of botnet victims

- Previous evaluations of botnet sizes based on distinct IPs may be grossly overestimated

- Botnet victims are users with poorly maintained machines and choose easily guessable passwords to protect sensitive data

- Interacting with registrars, hosting facilities, victim institutions, and law enforcement can be a complicated process 


\section{Credits}

UC Santa Barbara

- Brett Stone-Gross

- Marco Cova

- Lorenzo Cavallaro

- Bob Gilbert

- Martin Szydlowski

- Richard Kemmerer

- Chris Kruegel

- Giovanni Vigna

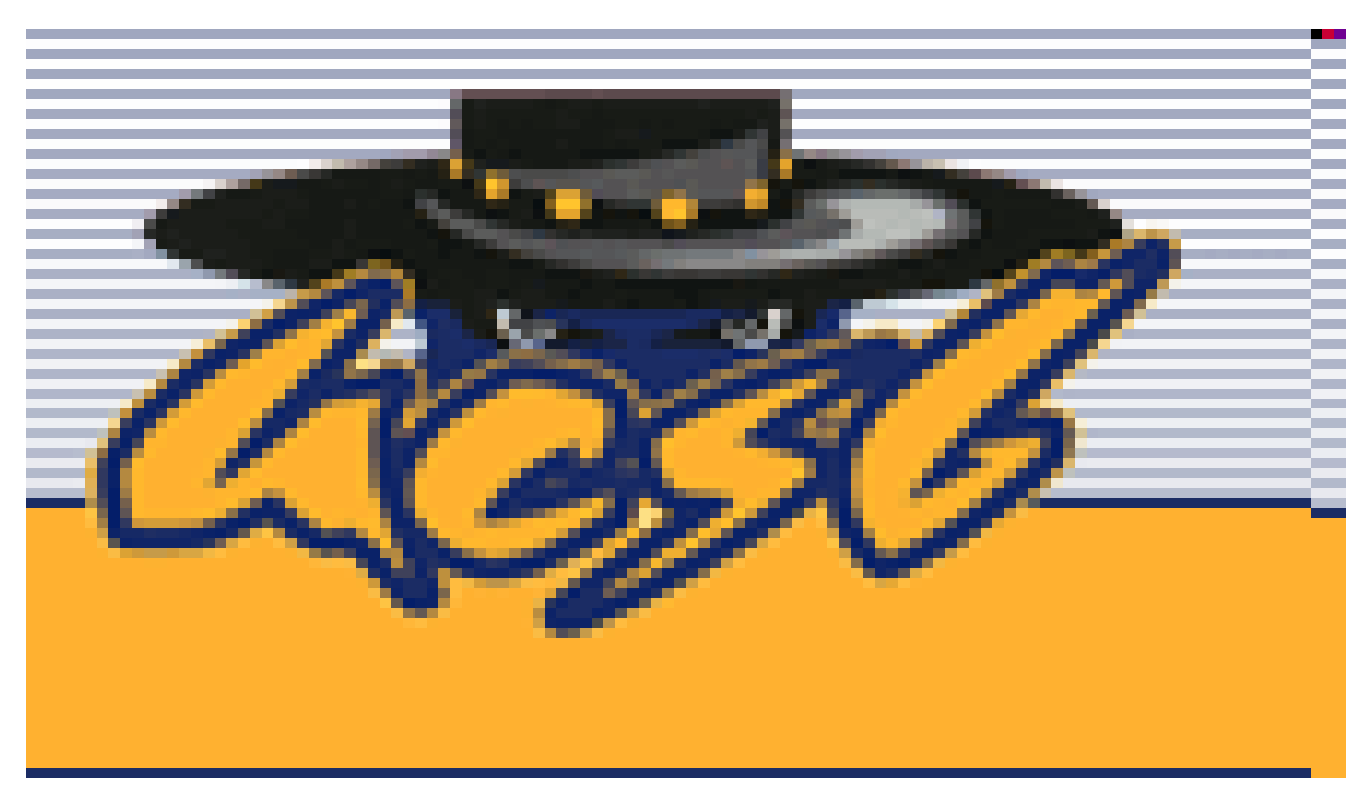




\section{Questions?}

UC Santa Barbara

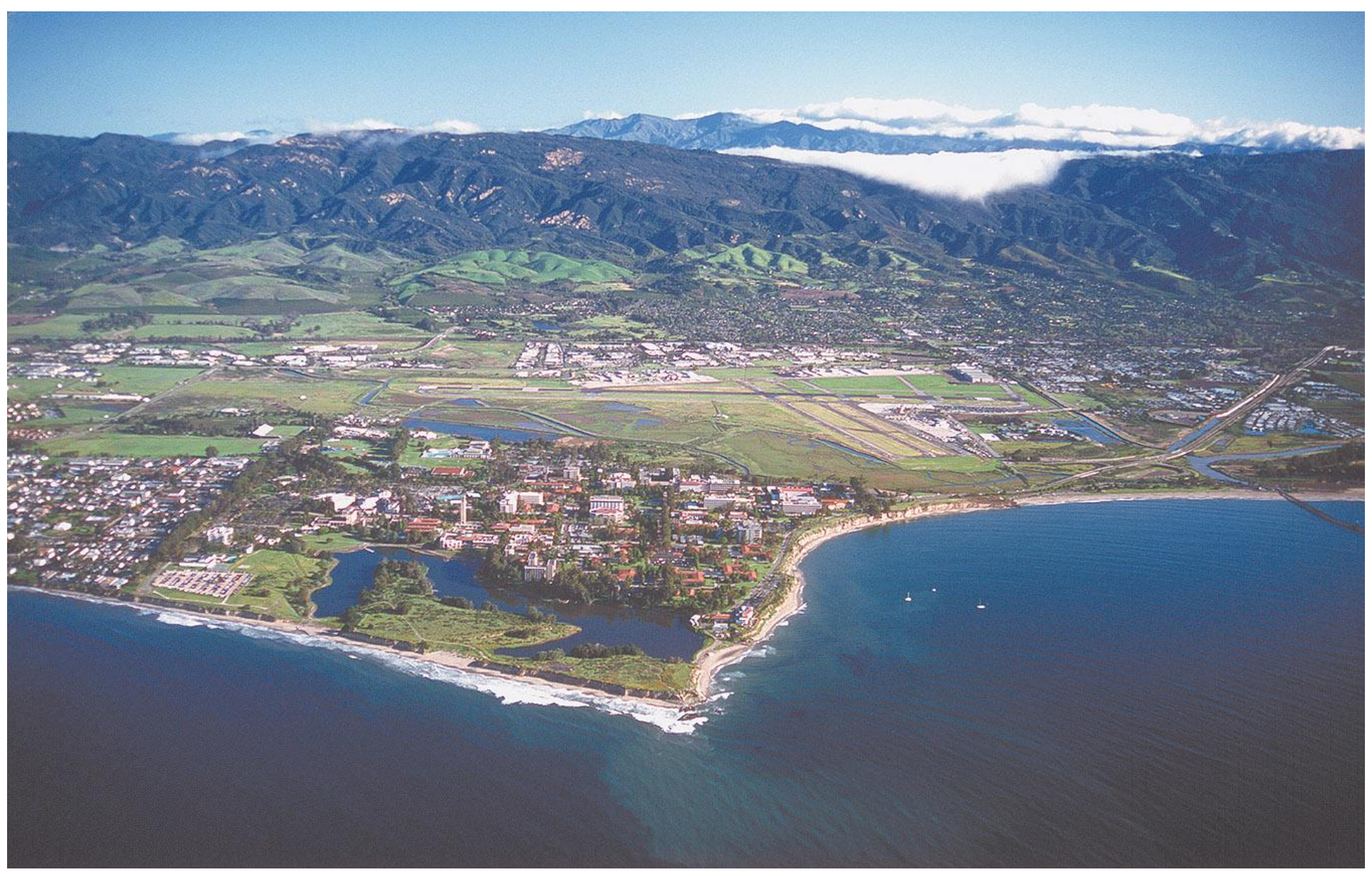

